\title{
The efficacy of iodine-125 interstitial brachytherapy for the treatment of locally advanced adenoid cystic carcinoma of the base of tongue: a non-surgical approach
}

\author{
Shuang Dong, MD!', Wei Li, MD², Yan Shi, MD!, Xiao-Ming Lv, MD!', Ming-Wei Huang, PhD, Assoc. Prof!!, \\ Prof. Jian-Guo Zhang, BS Med! \\ 'Department of Oral and Maxillofacial Surgery, Peking University School and Hospital of Stomatology, Beijing, China, ${ }^{2}$ Department of Oral \\ and Maxillofacial Surgery, Peking University School and Hospital of Stomatology, Beijing, China and Toyou Dental Clinic, Kunming, China
}

\begin{abstract}
Purpose: The present study retrospectively evaluated the effectiveness and safety of iodine-125 interstitial brachytherapy for treatment of primary locally advanced adenoid cystic carcinoma (ACC) of the base of tongue (BOT).

Material and methods: This study was a retrospective analysis of data of 19 patients with ACC, who were treated with iodine-125 interstitial brachytherapy between March 2008 and April 2018. Overall survival (OS), disease-free survival (DFS), local control (LC), and radiation-related toxicities were determined. Factors affecting the outcomes were evaluated.

Results: Six males and 13 females were included in our study, with a median follow-up time of 35 months. 3- and 5-year OS rates were $71.5 \%$ and $47.6 \%$, respectively. 3- and 5-year LC rates were $88.5 \%$ and $34.5 \%$, respectively. 3- and 5-year DFS rates were $54.7 \%$ and $21.9 \%$, respectively. A significant difference was observed in patients with or without brain metastases for OS rate. No severe acute toxicity was observed, while severe late toxicity was observed in one patient.

Conclusions: The results suggest that iodine-125 interstitial brachytherapy is an effective and safe option for the treatment of primary locally advanced ACC of the base of the tongue, with reasonably satisfactory LC and OS.

Key words: adenoid cystic carcinoma, interstitial brachytherapy, base of tongue, iodine-125.

\section{Purpose}

Adenoid cystic carcinoma (ACC) of the base of the tongue (BOT) is extremely rare, with only $8.8 \%$ of ACCs occurring in the head and neck region $[1,2]$. ACC is generally recognized by its typical characteristics of perineural invasion, indolent growth, late-onset local recurrence, and frequent distant metastases [3, 4]. The survival time of patients with early-stage ACCs is quite long due to slow growth pattern, but the long-term prognosis is relatively poor [5].

Radical resection with a negative margin combined with post-operative radiotherapy (RT) is the recommended treatment for ACC $[6,7]$. Because of high propensity for ACC infiltration into adjacent tissues, it is difficult to ensure a pathological negative margin, even with a wide resection [8]. Patients with locally advanced neoplasms located on the BOT, often undergo glossectomy and partial or total laryngectomy, which causes considerable swelling and speech function defects [9].

The outcome of locally advanced ACC is poor, with 3-year progress-free survival (PFS) and overall survival (OS) rates of $44-48 \%$ and $60-72 \%$, respectively [10, 11]. Chemotherapy, targeted therapy, and immunotherapy are used for patients with progressive and metastatic disease $[12,13]$, but objective responses are relatively low. Radiotherapy is one strategy for locally advanced tongue cancer, and it has the advantage of preserving tongue function compared to surgery combined with radiotherapy [14].

As a form of radiotherapy, iodine-125 ( $\left.{ }^{125} \mathrm{I}\right)$ interstitial brachytherapy (IBT) is used for the management of malignant neoplasms that occur in the salivary glands, especially locally advanced cases, with the advantage of 
organ preservation [15-17]. The present study retrospectively determined the efficacy and outcome of ${ }^{125} \mathrm{I}$ IBT in the treatment of locally advanced ACC of the BOT.

\section{Material and methods}

This was a retrospective study that was performed in accordance with international ethical standards and approved by the institutional review board. Nineteen patients who were pathologically diagnosed with ACC of the BOT treated with ${ }^{125}$ I interstitial brachytherapy were enrolled between March 2008 and April 2018. The median age was 56 years (range, 38 to 88 years). All patients $(100.0 \%)$ had primary tumors, and no previous treatment was performed. The most common major complaint was pain $(16 / 19,84.2 \%)$. Other symptoms included tongue movement limitation $(9 / 19,47.4 \%)$ and tongue numbness $(5 / 19,26.3 \%)$. According to the TNM $8^{\text {th }}$ staging edition of American Joint Committee on Cancer, all patients had stage T4aN0M0 disease. Table 1 shows the baseline characteristics of included patients. Surgery could not be performed in any patient due to local and systematic factors. Patients with distant metastases at diagnosis were excluded. All included patients provided written informed consent to receive ${ }^{125}$ I IBT, and agreed sharing their clinical data. None of the patients received external beam radiotherapy or chemotherapy. Half-life of ${ }^{125}$ I radioactive seeds was 59.4 days, and surface radioactivity was 18.5 to $25.9 \mathrm{MBq}$ per seed. Length and diameter were $45 \mathrm{~mm}$ and $0.8 \mathrm{~mm}$, respectively (model 6711, Beijing Atom and High Technique Industries Inc., Beijing, China). Gross tumor volume (GTV) was evaluated using computed tomography (CT), magnetic resonance imaging (MRI), or positron emission tomography (PET)/CT before treatment. Treatment plan was designed using DICOM format (treatment planning system, TPS, Beijing Atom and High Technique Industries Inc., Beijing, China), whereas clinical tumor volume (CTV) was defined as a $0-1.5 \mathrm{~cm}$ margin outside the tumor (the location where the tumor invaded the sur-

Table 1. Clinical characteristics of the 19 patients before ${ }^{125}$ I IBT for adenoid cystic carcinoma (ACC) of the base of the tongue

\begin{tabular}{lc} 
Characteristic & Number, $n(\%)$ \\
\hline Age (years) & \\
\hline$>56$ & $9(47.4)$ \\
\hline$\leq 56$ & $10(52.6)$ \\
\hline Sex & $6(31.6)$ \\
\hline Male & $13(68.4)$ \\
\hline Female & \\
\hline Symptom & $5(26.3)$ \\
\hline Paralysis & $9(47.4)$ \\
\hline Movement limitation & $17(89.5)$ \\
\hline Pain & $7(36.8)$ \\
\hline Maximum diameter $(\mathrm{cm})$ & $12(63.2)$
\end{tabular}

face of the BOT, or the surface of the pharynx was defined as $0 \mathrm{~cm})[18,19]$. Target volume was delineated and the prescribed dose ranged from 100 to $120 \mathrm{~Gy}$ in each patient. Organs at risk were then defined and included the parotid gland, submandibular gland, and skin, with a distance between organs at risk and $D_{90}$ isometric line of more than $1 \mathrm{~cm}$. Distribution of radioactive seeds was designed using TPS, and a three-dimensional (3D)-printed individual-puncture-guided template was designed. The needles were percutaneously punctured with sub-mandibular and sub-mental approaches. A 3D-printed individual template or CT navigation were used for implanting of ${ }^{125}$ I radioactive seeds under general anesthesia (Figure 1 ).

Computed tomography examination was performed within one week after the treatment to verify the number, position, and dosimetry parameters.

Regular follow-up was required, with a CT examination of the head and neck region performed every 6 months, or whenever necessary. MRI or PET/CT was done for further examination if required, and chest radiography was performed once annually. Tumor regression, local relapse, and distant metastasis were evaluated from clinical and imaging findings. Tumor regression was evaluated according to response evaluation criteria in solid tumors (RECIST), and complete response (CR), partial response (PR), progressive disease (PD), and stable disease (SD) were assessed. RT-associated toxicities were evaluated using the Radiation Therapy Oncology Group (RTOG) grading system. Probability of local recurrence, OS and DFS were calculated using Kaplan-Meier method, and associations between outcomes were determined with log-rank test using SPSS v.20.0 software. Statistical significance was defined as a $p$-value less than 0.05 .

\section{Results}

All patients successfully received ${ }^{125}$ I IBT treatment. The median number of ${ }^{125} \mathrm{I}$ seeds was 71 (range, 37-124 seeds), and the median number of needles was 19. For CT vitrification during the first week, the mean $\mathrm{D}_{90}$ was 116.8 $\pm 8.5 \mathrm{~Gy}$, which was higher than the prescribed dose in all patients. The mean $V_{100}$ was $92.0 \pm 1.3 \%$, and was greater than $90 \%$ in all cases. In addition, for all patients, $V_{150}$ was lower than $50 \%$, and $V_{200}$ was lower than $30 \%$. The homogeneity index (HI) ranged from $20 \%$ to $50 \%$, with the dose to organs at risk below the maximum limit.

Six months after the treatment, all patients had local remission, and swelling and speech functions improved to different degrees. The median follow-up time was 35.0 months (range, 13-98 months). At 6 months, 7 patients (36.8\%) had PR, 12 patients $(63.2 \%)$ had a CR, and no patients had SD or PD (Figure 2). Seven patients (36.8\%) experienced local recurrence, with an average recurrence time of 37.8 months (range, 13.5-58.5 months) since ${ }^{125}$ I IBT. Local failure occurred in 2 of these 7 patients within 2 years after the treatment (at 14 months and 24 months, respectively), and the other 5 patients had recurrent lesions after 3 years. Four patients did not receive treatment when local recurrence was detected due to old age, large size of the tumor, or the detection of brain metastases. Two patients received ${ }^{125}$ I IBT re-treatment, and 

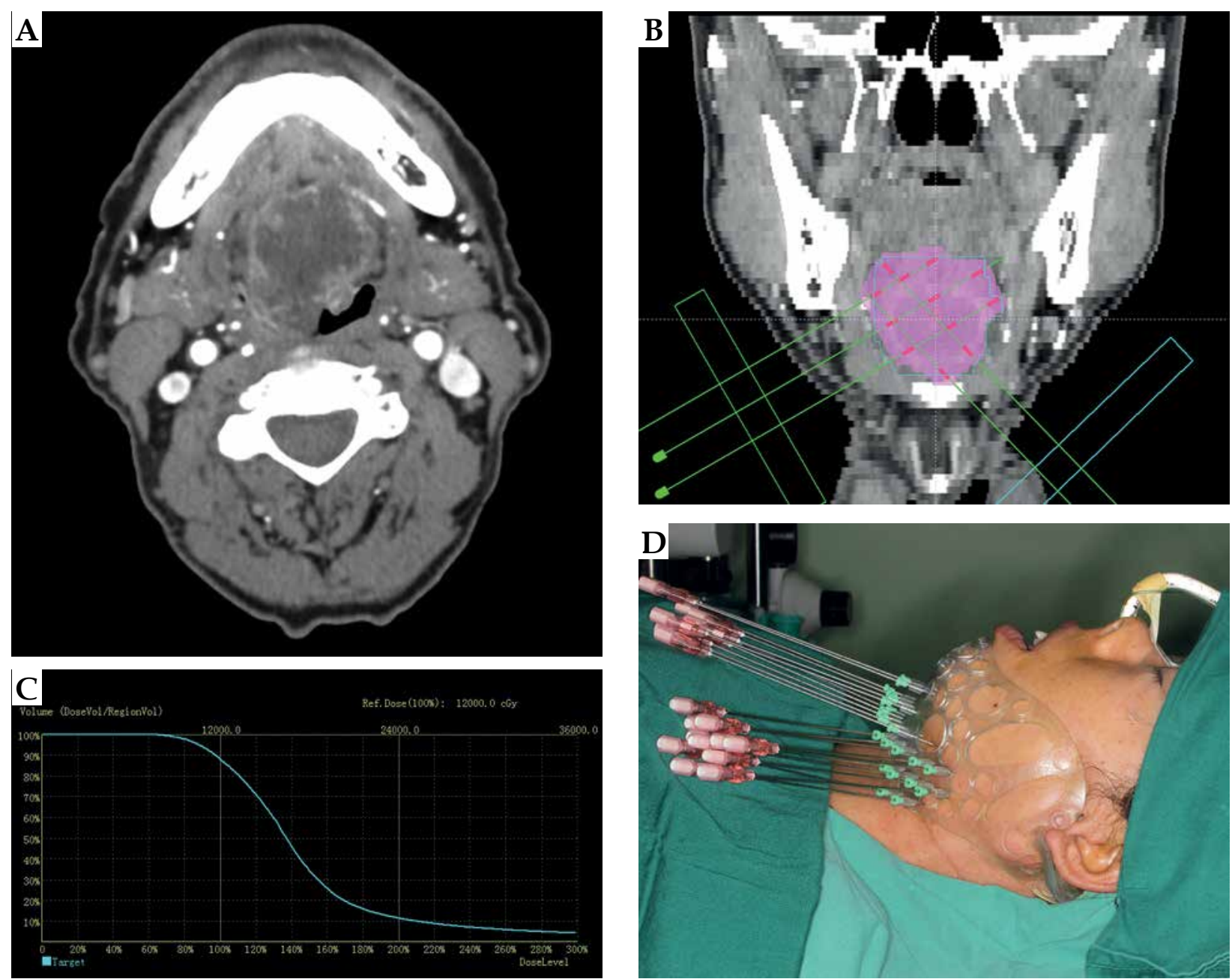

Fig. 1. A) Computed tomography (CT) image of a patient with adenoid cystic carcinoma (ACC) of the base of tongue (BOT); B) Clinical tumor volume (CTV) and the design of ${ }^{125}$ I radioactive seeds using TPS; C) Dose-volume histogram of CTV coverage (DVH) verification; D) 3D-printed individual template used during ${ }^{125}$ IBT

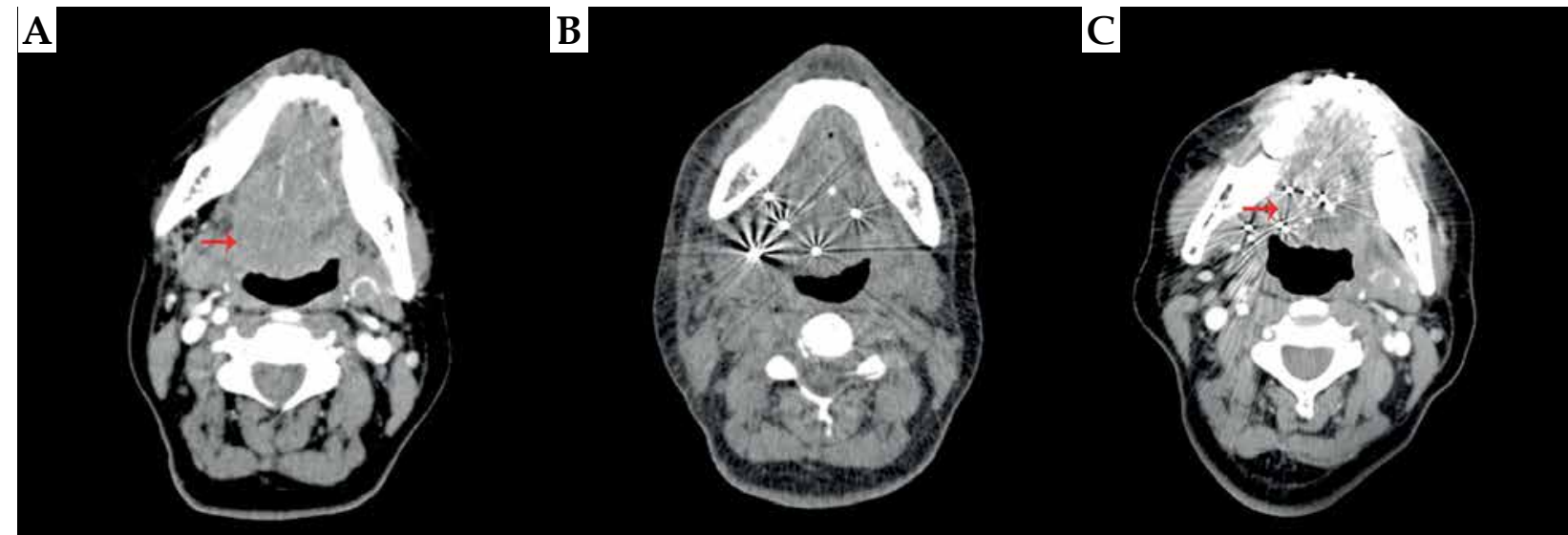

Fig. 2. A) Pre-operative computed tomography (CT) examination of a patient with locally advanced adenoid cystic carcinoma (ACC) of the base of tongue (BOT); B) Verified CT examination one day after ${ }^{125} \mathrm{I}$ IBT; C) Complete response three years after ${ }^{125}$ I IBT

1 patient underwent surgery. The 3- and 5-year local control (LC) rates calculated by Kaplan-Meier method were $88.5 \%$ and $34.5 \%$, respectively (Figure 3 ). The log-rank test revealed no significant differences in LC rate, based on age, sex, tumor size, distant metastasis, and matched peripheral dose.

Eight of the 19 patients died of the disease during follow-up period. Three patients $(15.8 \%)$ died of local 


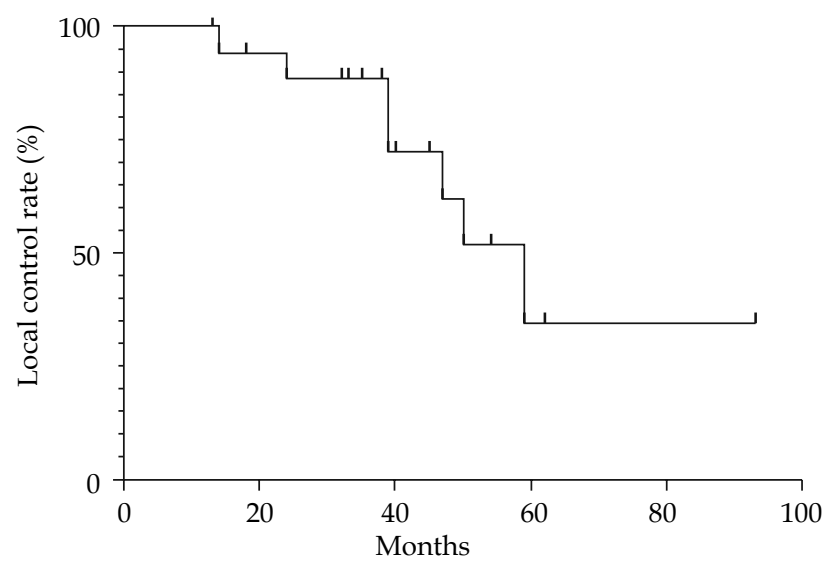

Fig. 3. Kaplan-Meier curve for local control (LC) rate

recurrence at 22,50 , and 59 months after ${ }^{125} \mathrm{I}$ IBT treatment (Table 2). Two patients $(10.5 \%)$ died of brain and lung metastases, and 2 patients $(10.5 \%)$ died of lung metastases. One patient died of hemorrhage after 18 months, with no local recurrence or distant metastasis detected. Another patient died from other causes. The 3- and 5-year OS rates were $71.5 \%$ and $47.6 \%$, respectively.

Neck lymph node metastases occurred in 2 patients $(10.5 \%)$, and 8 patients $(42.1 \%)$ had distant metastases (6 lung metastases, and 2 brain and lung metastases). The 3 - and 5-year DFS rates were $54.7 \%$ and $21.9 \%$, respectively (Figure 4).

For the 3-year OS rates in patients with distant metastases $(54.7 \%)$ and patients without it $(81.8 \%)$, a $p$-value of 0.321 was calculated using log-rank test, and no significant difference was observed. Two patients with brain metastases died at 13 and 47 months, which was a significant difference compared to the OS of other patients $(p=0.006,<0.5)$ (Figure 5).

All patients underwent ${ }^{125}$ I IBT successfully under general anesthesia. Tracheotomy was performed in 1 patient because of swelling of the BOT and the floor of the mouth. The other patients were able to breathe freely, and no serious complications, such as airway obstruction, were noted. Minor acute toxicities (RTOG grades 1 to 2 ) were observed in all patients, including mucositis

A

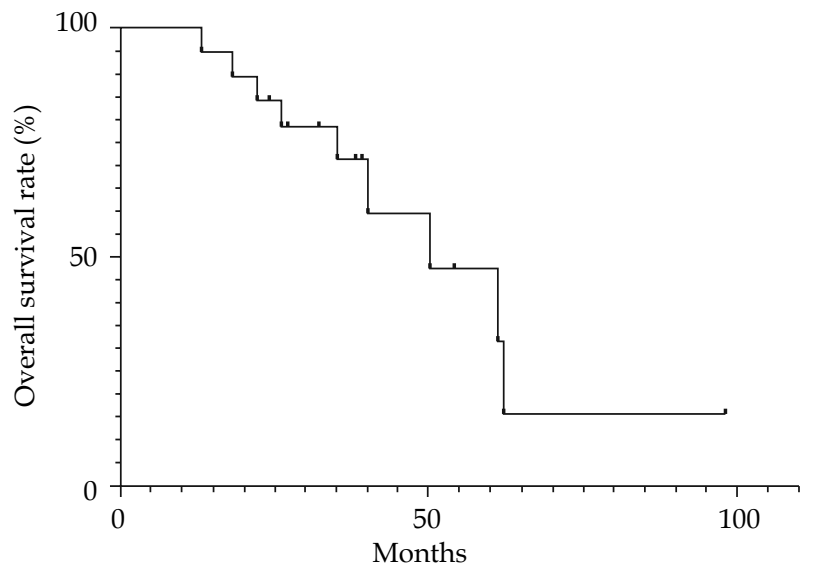

Table 2. Summary of treatment and outcomes for the 19 patients with adenoid cystic carcinoma (ACC) of the base of tongue (BOT)

\begin{tabular}{lc}
\hline Characteristic & Number, $n$ (\%) \\
\hline \begin{tabular}{l} 
Number of particles \\
\hline Median
\end{tabular} \\
\hline Range & 71 \\
\hline $\begin{array}{l}\text { Activity of particles (mCi) } \\
\leq 0.6\end{array}$ \\
\hline$>0.6$ & $8(42.1)$ \\
\hline Prescribed dose (Gy) & $11(57.9)$ \\
\hline$\leq 110$ & $11(57.9)$ \\
\hline$>110$ & $8(42.1)$ \\
\hline Local recurrence & $7(36.8)$ \\
\hline Yes & $12(63.2)$ \\
\hline No & $2(10.5)$ \\
\hline Neck lymph node metastasis & $17(89.5)$ \\
\hline Yes & $6(31.6)$ \\
\hline No & $2(10.5)$ \\
\hline Distant metastasis & $11(57.9)$ \\
\hline Lung only & $9(47.4)$ \\
\hline Lung and brain & $10(52.6)$ \\
\hline No & \\
\hline Death & \\
\hline Yes & \\
\hline No & \\
\hline
\end{tabular}

( $n=12$ patients, $63.2 \%$ ), erythema or dry desquamation ( $n=10$ patients, $52.6 \%$ ), and mild dysphagia or odynophagia ( $n=5$ patients, $26.3 \%$ ). No grade $3-4$ acute toxicities were observed. One patient (5.3\%) developed severe late toxicity. This patient had a hemorrhage 18 months after ${ }^{125}$ I IBT, and died due to airway obstruction, with no signs of local recurrence or distant metastasis. No other severe late toxicities (grades 3 to 4 ) were observed.

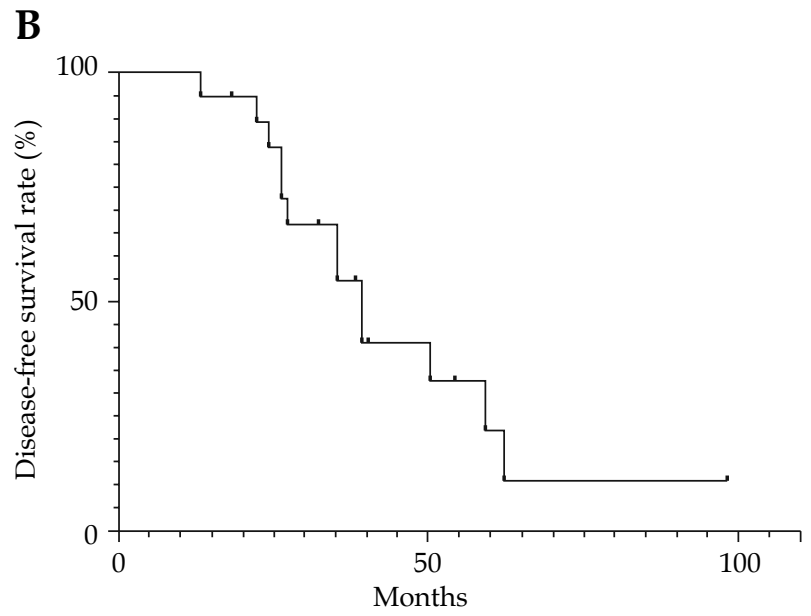

Fig. 4. A) Kaplan-Meier curve for overall survival (OS) rate; B) Kaplan-Meier curve for disease-free survival (DFS) rate 
A

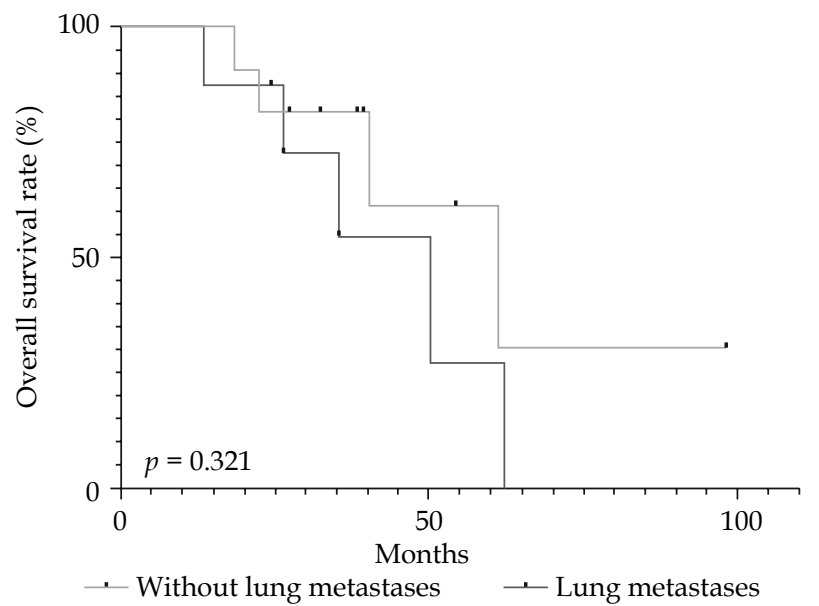

B

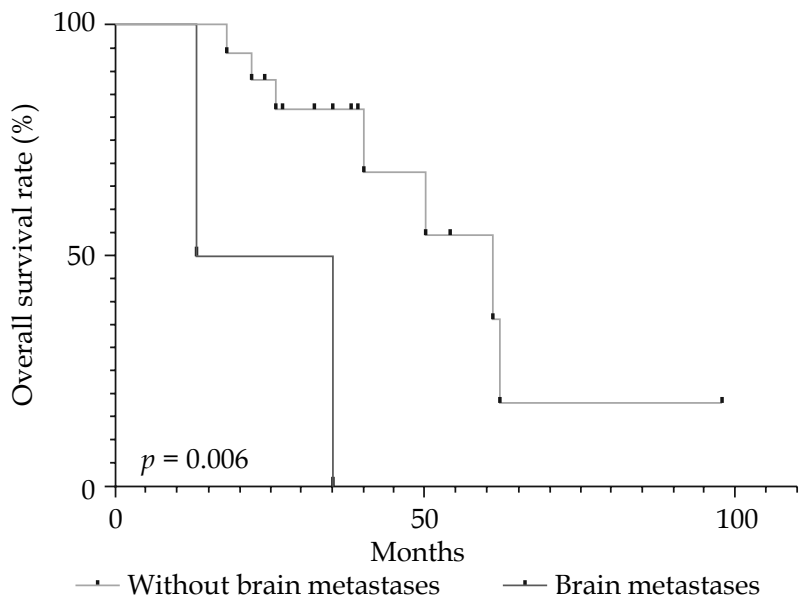

Fig. 5. Overall survival rate according to (A) lung metastases and (B) brain metastases using Kaplan-Meier analyses

\section{Discussion}

Primary ACC of the BOT is rare. Unlike mobile tongue, early detection and diagnosis is difficult for ACC of the BOT because of asymptomatic growth pattern and posterior site $[1,20]$. External beam radiotherapy (EBRT) plays an important role in the management of ACC. For early-stage cases, radical surgery combined with EBRT is the most common treatment. Mendenhall et al. concluded that OS rates of EBRT in ACC (5-year, 56\%; 10-year, $43 \%)$ were lower than surgery combined with EBRT (5-year, 94\%; 10-year, 91\%) [21]. Recent studies reported that 5 -year OS rates ranged from $59 \%$ to $74.8 \%$, and 5 -year LC rates ranged from $49 \%$ to $68.8 \%$ using proton radiotherapy (PRT) or carbon ion radiotherapy (CIRT) for head and neck ACC (HNACC) [10, 22-25].

However, the choice of surgery is controversial for locally advanced cases of ACC, especially of BOT. Quality of life decreased after wide surgical excision was performed [26]. As an alternative treatment, radiotherapy has advantages in function preservation. A 2009 German study reported 59 patients with inoperable or incompletely resected nasopharyngeal ACC, who were treated with bimodal RT consisting of intensity-modulated radiotherapy (IMRT) and carbon ion boost therapy (CIBT) [22]. Median follow-up time was 32 months, and 39 patients $(67 \%)$ were alive at the last follow-up. LC, DFS, and OS rates at 5 years were $49 \%, 54 \%$, and $69 \%$, respectively. These outcomes (LC, DFS, and OS) were better in patients with GTVs larger than $100 \mathrm{cc}$ than patients with GTVs smaller than $100 \mathrm{cc}$. There was a lower LC rate in patients with advanced disease (stage T4). The author concluded that adequate LC and OS rates were achieved using bimodal RT, and radiotoxicities were relatively mild. Another multi-center study in Japan included 289 inoperable patients, who were diagnosed with HNACC and treated with CIRT of 55.2 to 70.4 Gy [23]. 2-year OS, DFS, and LC rates were $94 \%, 68 \%$, and $88 \%$, respectively, and 5 -year OS, DFS, and LC rates were $74 \%, 44 \%$, and $68 \%$, respectively. The authors suggested that good LC and mild toxicity were achieved for HNACC patients treated with CIRT. Also, a study by Koto et al. treated 18 patients who were diagnosed with ACC of the BOT (including 17 advanced cases with stage T4b) using CIRT [27]. Reliable efficacy was achieved, with 5-year LC, DFS, and OS rates of $92 \%, 44 \%$, and $72 \%$, respectively (Table 3). Compared to IMRT, the use of PRT and CIRT was limited due to equipment availability, and patients had to bear the financial burden.

Brachytherapy is increasingly accepted as an alternative treatment for inoperable head and neck malignant neoplasms. High-dose brachytherapy and iridium-192 ( $\left.{ }^{192} \mathrm{Ir}\right)$ IBT were used for tongue squamous cell carcinoma [28-30]. To the best of our knowledge, no research on brachytherapy or ${ }^{125} \mathrm{I}$ was reported in ACC of the BOT. ${ }^{125}$ I IBT offers the advantages of minimal invasiveness, highly conformed delivery of high radiation dose to the target area, and a long half-life period. ${ }^{125}$ I IBT may be helpful in patients with inoperable diseases or poor general condition. $\mathrm{Xu}$ et al. reported 32 patients with local advanced ACC, involving the skull base treated with ${ }^{125} \mathrm{I}$ IBT alone [31]. 3-year OS and DFS rates were $62.6 \%$ and $46.4 \%$, respectively, with no severe acute RT reactions observed. Another research reported satisfying effects in 38 patients, who had a local failure or local advanced HNACC treated with ${ }^{125}$ I IBT [17]. Iodine- 125 radioactive seed IBT is an effective treatment for ACCs, which are locally advanced or endanger vital organs, with acceptable toxicity. The present study reported the 3-year LC, DFS, and OS rates of $88.5 \%, 54.7 \%$, and $71.5 \%$, respectively, and were similar to the outcomes of CIRT in the treatment of inoperable ACC. The 5-year LC, DFS, and OS rates in our study were $34.5 \%, 21.9 \%$, and $47.6 \%$, respectively, and were worse than the effect of PRT or CIRT [22-24, 27, 32]. However, due to low incidence of ACC of the BOT, more studies are needed to improve long-term efficiency of IBT, and comparisons between ${ }^{125}$ I IBT and external beam radiotherapy are required as well.

Local control failure is an important cause of death, and local recurrence rates ranged from $35 \%$ to $53 \%$ in patients with HNACC $[33,34]$. Akbaba reported 2- and 5 -year LC rates of $83 \%$ and $49 \%$, respectively, for nasopharynx ACC treated with IMRT and CIBT [22]. In an- 
Table 3. Summary of previous studies on locally advanced HNACC with radiotherapy

\begin{tabular}{|c|c|c|c|c|c|c|c|c|}
\hline Study [Ref.] & Year & Sites & $\begin{array}{c}\text { No. } \\
\text { of } \\
\text { patients }\end{array}$ & $\begin{array}{l}\text { Median } \\
\text { follow-up } \\
\text { (months) }\end{array}$ & Treatment & OS & LC & $\begin{array}{c}\text { Factors influencing } \\
\text { OS or LC }\end{array}$ \\
\hline Akbaba et al. [22] & 2019 & Nasopharynx & 59 & 32 & $\begin{array}{l}\text { IMRT with } \\
\text { CIRT boost } \\
\text { (72\% for } \\
\text { definite RT) }\end{array}$ & $\begin{array}{l}2 \text { years, } 87 \% \\
5 \text { years, } 69 \%\end{array}$ & $\begin{array}{l}2 \text { years, } 83 \% \\
5 \text { years, } 49 \%\end{array}$ & T4, GTV \\
\hline Sulaiman et al. [23] & 2018 & All & 289 & 30 & CIRT & $\begin{array}{l}2 \text { years, } 94 \% \\
5 \text { years, } 74 \%\end{array}$ & $\begin{array}{l}2 \text { years, } 88 \% \\
5 \text { years, } 68 \%\end{array}$ & $\begin{array}{c}\text { T stage, GTV, } \\
\text { performance status }\end{array}$ \\
\hline Ikawa et al. [24] & 2017 & All & 100 & 57 & CIRT & 5 years, $74.8 \%$ & 5 years, $68.6 \%$ & $\begin{array}{l}\text { Prescribed dose, } \\
\text { GTV, solid pattern }\end{array}$ \\
\hline Gentile et al. [36] & 2017 & Base of skull & 14 & 69 & PRT & 5 years, $59 \%$ & - & - \\
\hline Koto et al. [27] & 2016 & Base of tongue & 18 & 57 & CIRT & 5 years, $72 \%$ & 5 years, $92 \%$ & GTV \\
\hline
\end{tabular}

other study, 3- and 5-year LC rates were $100 \%$ in patients with ACC of the BOT treated with CIBT [27]. In our study, 7 patients were confirmed to have a recurrence, with the recurrence rate of $36.8 \%$. Two of these patients experienced a recurrence within 2 years, and other patients experienced a recurrence 3 years after ${ }^{125} \mathrm{I}$ IBT. The 3 - and 5 -year LC rates in our study were $88.8 \%$ and $36.0 \%$, respectively. Among the patients, who developed a recurrence, four did not receive further treatment, one received salvage surgery, and two received ${ }^{125}$ I IBT re-treatment. These results suggest that ${ }^{125} \mathrm{I}$ IBT is suitable for locally advanced ACC of the BOT, with acceptable LC and survival. Iodine-125 IBT may also be used as a re-treatment in patients with recurrent lesions.

However, the improper handling of tumors of the BOT may lead to severe complications, including airway obstruction. EBRT may produce acute or late radiation-induced toxicities, and severe complications may occur, such as osteoradionecrosis and soft tissue necrosis $[27,35]$. Koto et al. reported 2 occurrences of osteonecrosis of the jaw and 1 occurrence of hemorrhage among 18 patients with ACC of the BOT [27]. In the current study, ${ }^{125}$ I IBT was performed as a one-time procedure, in contrast to external beam radiotherapy. Relatively mild complications occurred in the patients who received ${ }^{125} \mathrm{I}$ IBT compared to those who received CIBT or IMRT. No acute airway obstruction was observed while using the precisely designed individual templates and CT guidance. During the follow-up period, one patient experienced hemorrhage and died at 18 months, with no sign of local recurrence or distant metastasis. The hemorrhage may have been due to an extensive original tumor invasion and large target area. No severe complications were observed in other patients. Our results suggest that ${ }^{125} \mathrm{I}$ IBT is minimally invasive and relatively safe for the treatment of ACC of the BOT. We should focus on respiratory conditions when GTV is relatively large.

Overall survival rate is significantly influenced by distant metastases, and $70 \%$ of patients' deaths due to ACC were caused by lung metastases [8]. In our study, the 3-year OS rates of patients with and without lung metastases were $54.7 \%$ and $81.8 \%(p>0.05)$, respective- ly. However, two patients with brain metastases died at 13 and 35 months, and brain metastases significantly influenced the OS rate $(p=0.032)$.

Our study has limitations because the sample size was relatively small. Firstly, the clinico-pathological characteristics and statistical analysis partially reflect this objective fact. Secondly, our follow-up period was relatively short. To our knowledge, ACC may recur after decades. Thirdly, in several patients, GTV was defined by multiple imaging, which could lead to inconsistency in the delineation of the target area. Further studies of ${ }^{125} \mathrm{I}$ IBT efficiency and toxicity, with longer follow-up periods are needed.

\section{Conclusions}

In conclusion, ${ }^{125} \mathrm{I}$ IBT is an option for the treatment of ACC of the base of the tongue, and provides relatively satisfactory LC and OS rates, with acceptable toxicities. Iodine-125 IBT is minimally invasive, conformal, and to some degree, prevents severe complications caused by surgery or EBRT. However, delineation of the tumor target area and matched peripheral dose demand further studies.

\section{Disclosure}

The authors report no conflict of interest.

\section{References}

1. Han N, Ong H, Liu Z et al. Lymph node involvement predicts poor prognosis in primary tongue adenoid cystic carcinoma: A preliminary study of 54 cases. J Craniomaxillofac Surg 2017; 45: 589-594.

2. Kirke DN, Chitguppi C, Rubin SJ et al. Adenoid cystic carcinoma of the base of tongue: A population-based study. Am J Otolaryng 2017; 38: 279-284.

3. van Weert S, Reinhard R, Bloemena E et al. Differences in patterns of survival in metastatic adenoid cystic carcinoma of the head and neck. Head Neck 2017; 39: 456-463.

4. Chang CF, Hsieh MY, Chen MK et al. Adenoid cystic carcinoma of head and neck: A retrospective clinical analysis of a single institution. Auris Nasus Larynx 2018; 45: 831-837.

5. Ouyang DQ, Liang LZ, Zheng GS et al. Risk factors and prognosis for salivary gland adenoid cystic carcinoma in south- 
ern China: A 25-year retrospective study. Medicine (Baltimore) 2017; 96: e5964.

6. Mannelli G, Cecconi L, Fasolati M et al. Parotid adenoid cystic carcinoma: Retrospective single institute analysis. Am J Otolaryngol 2017; 38: 394-400.

7. Pfister DG, Spencer S, Adelstein D, et al. Head and neck cancers, Version 2.2020, NCCN Clinical Practice Guidelines in Oncology. J Natl Compr Canc Netw 2020; 18: 873-898.

8. Takebayashi S, Shinohara S, Tamaki H et al. Adenoid cystic carcinoma of the head and neck: a retrospective multicenter study. Acta Otolaryngol 2018; 138: 73-79.

9. Machtay M, Perch S, Markiewicz D et al. Combined surgery and postoperative radiotherapy for carcinoma of the base of radiotherapy for carcinoma of the base of tongue: analysis of treatment outcome and prognostic value of margin status. Head Neck 1997; 19: 494-499.

10. Dautruche A, Bolle S, Feuvret L et al. Three-year results after radiotherapy for locally advanced sinonasal adenoid cystic carcinoma, using highly conformational radiotherapy techniques proton therapy and/or tomotherapy. Cancer Radiother 2018; 22: 411-416.

11. Chopra S, Kamdar DP, Cohen DS et al. Outcomes of nonsurgical management of locally advanced carcinomas of the sinonasal cavity. Laryngoscope 2017; 127: 855-861.

12. Dillon PM, Petroni GR, Horton BJ et al. A phase II study of dovitinib in patients with recurrent or metastatic adenoid cystic carcinoma. Clin Cancer Res 2017; 23: 4138-4145.

13. Kokkali S, Ntokou A, Drizou M et al. Nivolumab in patients with rare head and neck carcinomas: A single center's experience. Oral Oncol 2020; 101: 104359.

14. Park S, Cho Y, Lee J et al. Survival and functional outcome after treatment for primary base of tongue cancer: a comparison of definitive chemoradiotherapy versus surgery followed by adjuvant radiotherapy. Cancer Res Treat 2018; 50: 1214-1225

15. Zheng L, Lv X, Shi Y et al. (125)I interstitial brachytherapy for the treatment of myoepithelial carcinoma of the oral and maxillofacial region. Brachytherapy 2016; 15: 240-245.

16. Liu SM, Wang HB, Sun Y et al. The efficacy of iodine- 125 permanent brachytherapy versus intensity-modulated radiation for inoperable salivary gland malignancies: study protocol of a randomised controlled trial. BMC Cancer 2016; 16: 193.

17. Huang MW, Zheng L, Liu SM et al. 125I brachytherapy alone for recurrent or locally advanced adenoid cystic carcinoma of the oral and maxillofacial region. Strahlenther Onkol 2013, 189: 502-507.

18. Chen P, Wu WJ, Yi ZQ et al. (125) I interstitial brachytherapy in management of pediatric skull base tumors. Pediatr Blood Cancer 2019; 66: e27622.

19. Wu ZY, Wu WJ, Zheng L et al. Efficacy of combined surgery and (125) I seed brachytherapy for treatment of primary mucoepidermoid carcinoma of the parotid gland. Head Neck 2019; 41: 3219-3225.

20. Luna-Ortiz K, Carmona-Luna T, Cano-Valdez AM et al. Adenoid cystic carcinoma of the tongue - clinicopathological study and survival analysis. Head Neck Oncol 2009; 1: 15.

21. Mendenhall WM, Morris CG, Amdur RJ et al. Radiotherapy alone or combined with surgery for adenoid cystic carcinoma of the head and neck. Head Neck 2004; 26: 154-162.

22. Akbaba S, Ahmed D, Lang K et al. Results of a combination treatment with intensity modulated radiotherapy and active raster-scanning carbon ion boost for adenoid cystic carcinoma of the minor salivary glands of the nasopharynx. Oral Oncol 2019; 91: 39-46.

23. Sulaiman NS, Demizu Y, Koto M et al. Multicenter study of carbon-ion radiation therapy for adenoid cystic carcinoma of the head and neck: subanalysis of the Japan Carbon-Ion Ra- diation Oncology Study Group (J-CROS) Study (1402 HN). Int J Radiat Oncol Biol Phys 2018; 100: 639-646.

24. Ikawa $\mathrm{H}$, Koto M, Takagi R et al. Prognostic factors of adenoid cystic carcinoma of the head and neck in carbon-ion radiotherapy: The impact of histological subtypes. Radiother Oncol 2017; 123: 387-393.

25. Jensen AD, Poulakis M, Nikoghosyan AV et al. High-LET radiotherapy for adenoid cystic carcinoma of the head and neck: 15 years' experience with raster-scanned carbon ion therapy. Radiother Oncol 2016; 118: 272-280.

26. Balbinot J, Real CS, Melo CC et al. Quality of life in tongue cancer treated patients before and after speech therapy: a randomized clinical trial. Braz J Otorhinolaryngol 2020; S1808-8694(20)30194-4.

27. Koto M, Hasegawa A, Takagi R et al. Evaluation of the safety and efficacy of carbon ion radiotherapy for locally advanced adenoid cystic carcinoma of the tongue base. Head Neck 2016, 38: E2122-E2126

28. Santos MA, Guinot JL, Tortajada MI et al. High-dose-rate interstitial brachytherapy boost in inoperable locally advanced tongue carcinoma. Brachytherapy 2017; 16: 1213-1218.

29. Takacsi-Nagy Z, Oberna F, Koltai P et al. Long-term outcomes with high-dose-rate brachytherapy for the management of base of tongue cancer. Brachytherapy 2013; 12: 535-541.

30. Johansson B, Karlsson L, Reizenstein J et al. Pulsed dose rate brachytherapy as the boost in combination with external beam irradiation in base of tongue cancer. Long-term results from a uniform clinical series. J Contemp Brachytherapy 2011; 3: 11-17.

31. Xu N, Zheng L, Wu WJ et al. Definitive (125)I brachytherapy of locally advanced adenoid cystic carcinoma involving the skull base with satisfying efficacy and safety. J Oral Maxillofac Surg 2019; 77: 2143-2153.

32. Wolkow N, Jakobiec FA, Lee H et al. Long-term outcomes of globe-preserving surgery with proton beam radiation for adenoid cystic carcinoma of the lacrimal gland. Am J Ophthalmol 2018; 195: 43-62.

33. Oplatek A, Ozer E, Agrawal A et al. Patterns of recurrence and survival of head and neck adenoid cystic carcinoma after definitive resection. Laryngoscope 2010; 120: 65-70.

34. Marcinow A, Ozer E, Teknos T et al. Clinicopathologic predictors of recurrence and overall survival in adenoid cystic carcinoma of the head and neck: a single institutional experience at a tertiary care center. Head Neck 2014; 36: 1705-1711.

35. Christopherson K, Reisman DN, Fredenburg K et al. Base of tongue squamous cell carcinoma with infiltrative bone marrow carcinomatosis after definitive chemoradiation: A case report. Head Neck 2016; 38: E2449-E2453.

36. Gentile MS, Yip D, Liebsch NJ et al. Definitive proton beam therapy for adenoid cystic carcinoma of the nasopharynx involving the base of skull. Oral Oncol 2017; 65: 38-44. 